\title{
Highlights
}

- A maximal number of factual claims with credibility higher than the precision requirement are extracted from the Web.

- The learning model is up to 20 times faster than traditional learning.

- The proposed model extracts up to 6 times more highly credible factual claims than a typical information extraction process.

- The proposed model requires less than 57\% label information to extract the same number of highly credible factual claims.

- The proposed model is robust to $20 \%$ noisy dáta with only $6 \%$ deviation.

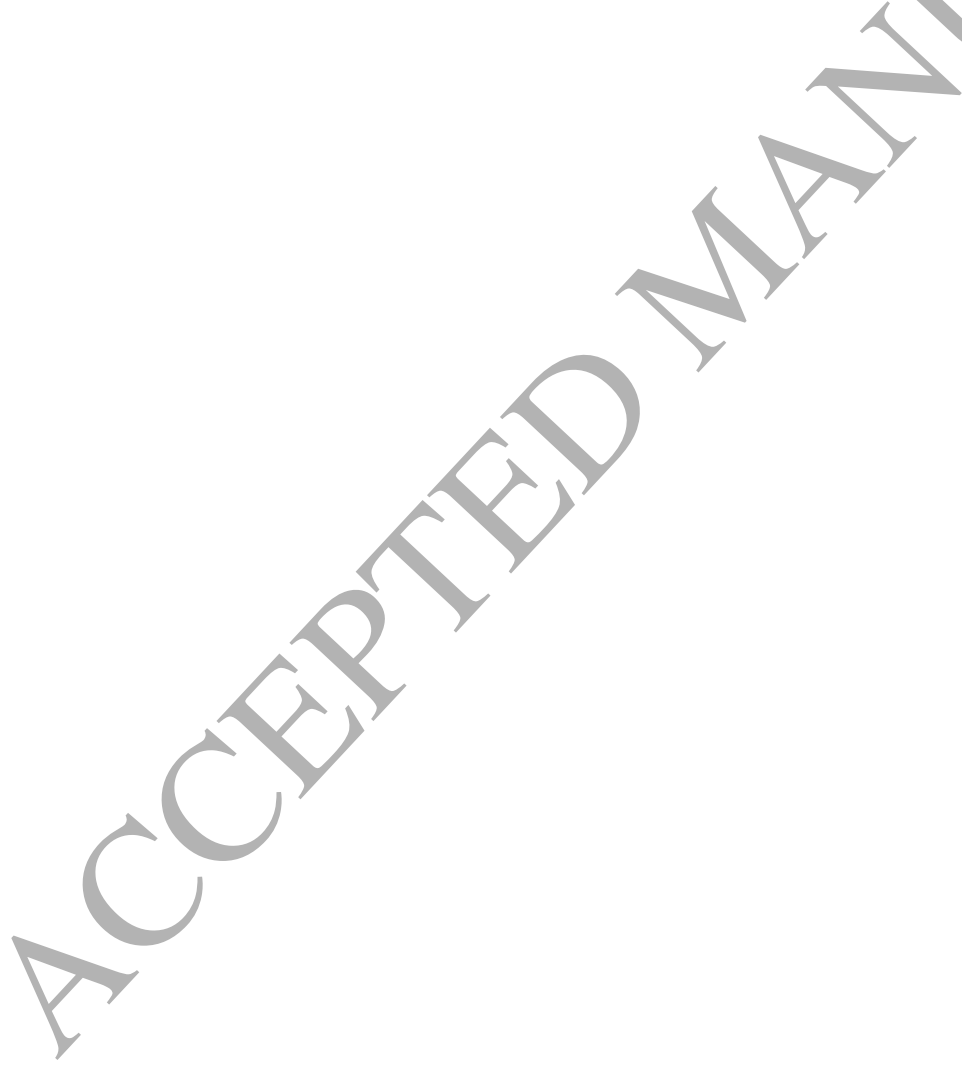




\section{Maximal Fusion of Facts on the Web with Credibility Guarantee}

Nguyen Thanh Tam ${ }^{\mathrm{a}}$, Phan Thanh Cong ${ }^{\mathrm{a}}$, Nguyen Quoc Viet Hung ${ }^{\mathrm{b}}$, Karl Aberer $^{\mathrm{a}}$, Bela Stantic ${ }^{\mathrm{b}}$

${ }^{a}$ École Polytechnique Fédérale de Lausanne, Switzerland

${ }^{b}$ Griffith University, Australia

\section{Abstract}

The Web became the central medium for valuable sources of information fusion applications. However, such user-generated resources are often plagued by inaccuracies and misinformation as a result of the inherent openness and uncertainty of the Web. While finding objective data is non-trivial, assessing their credibility with a high confidence is even harder due to the conflicts of information between Web sources. In this work, we consider the novel setting of fusing factual data from the Web with a credibility guarantee and maximal recall. The ultimate goal is that not only the information should be extracted as much as possible but also its credibility must satisfy a threshold requirement. To this end, we formulate the problem of instantiating a maximal set of factual information such that its precision is larger than a pre-defined threshold. Our proposed approach is a learning process to optimize the parameters of a probabilistic model that captures the relationships between data sources, their contents, and the underlying factual information. The model automatically searches for best parameters without pre-trained data. Upon convergence, the parameters are used to instantiate as much as factual information with a precision guarantee. Our evaluations of real-world datasets show that our approach outperforms the baselines up to 6 times.

Keywords: information fusion, knowledge extraction, precision guarantee, probabilistic model, credibility analysis 


\section{Introduction}

The Web became the central medium for valuable sources of information such as articles, blogs, and wikis, where people constantly share knowledge, report scientific studies, upload comments, and write reviews. As a consequence, the

5 Web emerged as the prime source for extracting and fusing information $[1,2,3]$. Applications that benefit from information fusion on top of the Web include systems for knowledge base [4], decision-support [5], and recommendations [6].

However, the Web is often plagued by unreliable and untrustworthy information as Web users can post their contents freely. The openness of the Web have enabled data sources to share their information and relay data without proper attribution. Moreover, the sources also have wide-ranging levels of quality, as the content is generated by human. This leads to potential erroneous, inconsistent, and out-of-date information when extracting data from the Web. While the quality of Web data will be still questionable in future, it provides 15 a unique opportunity to fuse the wisdom of the crowd into factual information $[7,8,9,10,11]$.

As a result, there is a need of identifying factual information out of Web data. That is, we should extract the data of high credibility and filter out uncertain and erroneous information. This is motivated by the fact that nowadays there 20 are application domains that need extremely high-quality information such as medicine and politics, where the extracted data is sensitive and affects many lives.

However, existing works on information extraction mainly focus on extracting syntactical properties of data such as unstructured contents on the Web [12]. Factual information is hindered by the sheer amount of available Web data and its unstructured, free-text representation. The literature concerns how to parse these unstructured data effectively, meaning the data should be correctly identified as-is by avoiding parsing errors. As a result, the extracted data is often structured and believed to be credible in terms of error-free extraction. In fact, 30 the resulting data only comes with high recall of information coverage, but still 
questionable in terms of quality due to the inherent uncertainty of the Web. Even though there are a few body of work that study the quality assessment of data as a post-processing step [13], the output of their quality classifiers is still unpredictable even when more training data is provided. In other words, 35 we do not know exactly how much expert knowledge is needed or whether it is possible to reach a certain quality threshold.

Going beyond the literature, we propose an information fusion framework that comes with a credibility guarantee. Our model allows end-user to specify a credibility threshold as input. Then, it tries to extract and aggregate factual

40 information from Web data as much as possible while ensuring the overall credibility of extracted information is larger than the pre-defined threshold. In other words, we overcome the inherent trade-off between the precision of information credibility and the recall of information coverage in a novel way for sensitive applications: maximizing the recall while preserving the precision at least better 45 or equal to a pre-defined requirement.

In summary, the contributions of this paper are as follows:

- Problem: We formally define a novel problem, namely credibility extraction, of extracting structured knowledge from the Web with a credibility guarantee. The problem can be formulated on top of any generic information extraction frameworks.

- Model: We propose a joint probabilistic model for credibility extraction. The model captures not only the mutual relationship between structured knowledge, unstructured Web contents and data sources but also the individual features that indicates the level of credibility.

- Method: We propose a credibility reinforcement algorithm that allows the credibility guarantee to be met while achieving largest output size.

- Applications: We demonstrate the applicability of our method in healthcare, politics, and social domains. 
The remainder of this paper is organized as follows. In $\S 2$, we formally define 60 the problem of credibility extraction. We then introduce how to model various credibility elements using the factor graph model in $\S 3$. In $\S 4$, we discuss a reinforcement algorithm to learn the model that satisfies the credibility constraint. In $\S 7$, we provide empirical analysis of our approach using real-world datasets. Finally, we provide the related works in $\S 8$ before $\S 9$ concludes the paper.

\section{2. Model and Problem Statement}

\subsection{Model}

While our setting is generic for any information fusion framework, we frame our problem in the context of factual knowledge extraction by fusing data from multiple sources $[14,15,16]$. We model the setting of factual knowledge extraction by a set of data sources $S=\left\{s_{1}, \ldots, s_{u}\right\}$ and a set of documents $D=\left\{d_{1}, \ldots, d_{m}\right\}$. A source could be a user, a website, a news provider, or a business entity. It provides multiple documents, each of which is often textual (e.g., a tweet, a news item, a web page, or a forum post). Claims can be extracted from documents by NLP techniques [17]. A claim can be supported or refuted by multiple documents, i.e. the factual value of the claim is still not a consensus and needs to be computed from these document-claim links. We denote the set of all unique claims, or candidate facts, as $C=\left\{c_{1}, \ldots, c_{n}\right\}$.

Example 1. In the snopes dataset [18], the claim Eating turkey makes people especially drowsy is mentioned in several webpages of multiple sources such as earthsky.org ${ }^{1}$, www.webmd.com ${ }^{2}$, kidshealth.org ${ }^{3}$, etc. The factual value of the claim is false (non-credible) as being examined by the Snopes editor ${ }^{4}$.

Sources, documents, and claims jointly represent a factual networked data, denoted by a tuple $N=\langle S, D, C\rangle$. To construct an accurate knowledge base,

\footnotetext{
${ }^{1}$ http://earthsky.org/human-world/does-eating-turkey-make-you-sleepy

${ }^{2}$ http://www. webmd.com/food-recipes/the-truth-about-tryptophan

${ }^{3}$ http://kidshealth.org/en/kids/turkey-sleepy.html

${ }^{4}$ wWw.snopes.com/food/ingredient/turkey.asp
} 
we need to potentially infer labels for each claim $c$ in $C$, where $c_{i}=1$ denotes 85 that the claim is credible, whereas $c_{i}=0$ represents the opposite.

Features. A networked data is constructed based on sources and documents, each encoded using a set of features, such as the frequency of updates at a source or linguistic characteristics of a document. Our model abstracts from the specific nature of these features, but takes into account that the trustworthiness of a 90 source and the language quality of a document often have a strong influence on the credibility of the extracted claims. Each source $s_{k}$ is associated with a feature vector $\left\langle I_{1}^{S}\left(s_{l}\right), \ldots, I_{m_{S}}^{S}\left(s_{l}\right)\right\rangle$ of $m_{S}$ source features. In the same vein, $\left\langle I_{1}^{d}\left(d_{j}\right), \ldots, I_{m_{D}}^{d}\left(d_{j}\right)\right\rangle$ is a vector of $m_{D}$ document features assigned to each document $d_{j}$.

95 Relations. Features of sources and documents interact with each other, as well as the credibility of the extracted claims, so that we consider the following relations between them:

- Causal relation: Claims are provided in a document by a source. Thus, a claim's credibility depends on both trustworthiness of the source and the language quality of the document. A claim is more likely to be credible, if it is posted by a trustworthy source using confident and objective language. Yet, the intentions of a source, and thus its trustworthiness, may change over different contexts and hence documents.

- Mutual relation: Causal relations are complemented by mutual relations that stem from the overlap between causal relations. Multiple documents, potentially from multiple sources, may contain the same claim. For example, if a source disagrees with a claim that is accepted by several sources, it shall be regarded as not trustworthy.

To give the intuition of networked data, we consider the scenario of Web data with two providers $s_{1}$ and $s_{2}$. Each provider runs a website domain, which contains three webpages $d_{1}, d_{2}$, and $d_{3}$. These webpages are overlapping with each other in terms of claims $c_{1}, c_{2}$, and $c_{3}$. Taking the real-world forms of the above mentioned providers as a representation of the underlying networked 
data, a simplified view on a factual dataset is shown in Fig. 1.

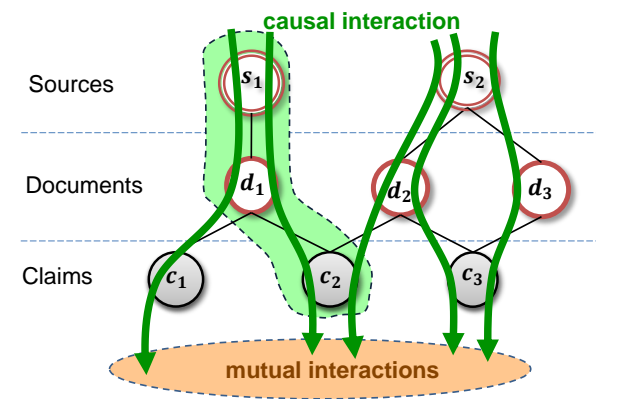

Figure 1: A fraction of factual networked data

\subsection{Problem Statement}

Our overall goal is to construct a credible knowledge extractor with a precision guarantee. In particular, our algorithm allows user to specify a precision threshold as input. We call this problem credibility extraction or knowledge extraction with credibility guarantee.

Problem 1 (Credibility Extraction). Given a factual networked data $N=$ $\langle S, D, C\rangle$, where $S$ is a set of data sources, $D$ is a set of their documents, and $C$ is a set of distinct claims, and a precision threshold $\tau$, return a set of claims considered to be credible with precision at least $\tau$ that maximizes output size.

$$
R^{*}=\underset{R \subseteq C, \operatorname{Prec}(R) \geq \tau}{\arg \max }|R|
$$

where $\operatorname{Prec}(R)$ is the ratio of true positives over the size of $R$ itself. It should be emphasized that our problem is orthogonal to the traditional parameter estimation [19], which does not give a fine-grained control over the precision and the output size is often unpredictable. In other words, the traditional setting aims to maximizes the precision and often scarifies recall, our setting 125 allows user to control this trade-off by specifying the precision threshold.

Our problem assumes an access to an oracle, who can labeled a claim $c$ as credible or non-credible, meaning that the precision $\operatorname{Prec}(R)$ can be computed 
at any time. This assumption matches with real-life settings, e.g. training data, prior knowledge, crowdsourcing, human expert [11, 20, 21, 22, 23]. Note that the output size is also known as recall since the size of ground truth is fixed. Solving this problem needs to take into account the labeling cost - the number of labels that we need from the oracle - that we seek to minimize. Here the computation time - the time it takes to produce an output - is neglected since our problem concerns the quality of the output. Moreover, thanks to the efficient self-configuration of our proposed probabilistic model using factor graph below (e.g. using sampling), the computation is scalable.

\section{Modeling Credibility on the Web}

Construction of a set of credible facts requires the computation of probabilities: for each claim $c$, we need to determine the probability of its being credible, i.e. $\operatorname{Pr}(c)$. In the context of this work, therefore, we ground the computation of probabilities for claims in the individual features and relations defined for the networked data. From this starting point, we adopt a model in which a claim is a random variable. Then, relations express dependencies between these random variables and features are evidence for their truth values. Below, we first show how this idea is formalized using the model of a factor graph, before we turn to the actual computation of probabilities for the claims.

\subsection{Creation of the factor graph}

We capture the dependencies between the random variables of claims, features, and relations by means of a probabilistic graphical model, namely a factor graph. In general, a factor graph establishes a relation between functions (called factors) that are defined over subsets of random variables. The model enables self-configuration when new information becomes available, which is an important asset to support information extraction: With the arrival of new Web data, or candidate facts, the model is updated incrementally by adding variables and 155 factors. 
A factor graph is a bipartite graph $M=\langle V, F, E\rangle$ where $V$ is a set of random variables or evidence, $F$ is a set of functions (factors), and $E \subseteq\{\{v, f\} \mid v \in$ $V, f \in F\}$ are undirected edges. A set of random variables $V$ and a set of factors $F$ fully characterizes a factor graph. The definition of the edges relates each factor $f\left(v_{1}, \ldots, v_{e}\right) \in F$ to the random variables over which it is defined, i.e., $\left\{f, v_{i}\right\} \in E$ for $v_{i} \in V, 1 \leq i \leq e$.

In our context, there are three types of random variables representing claims, sources, and documents. We overload notation and use $C, S$, and $D$ to refer to the actual claims, sources, and documents, as well as the associated random variables, i.e., $V=C \cup S \cup D$ defines the variable nodes of the factor graph. Further, the model includes claim factors $F_{C}$, source factors $F_{S}$, and document factors $F_{D}$ to encode relations between the variables, i.e. $F=F_{C} \cup F_{S} \cup F_{D}$ defines the factor nodes of the factor graph.

Source Variables. We denote $s_{i} \in\{0,1\}$ as the random variable that represents the source trustworthiness. In that, $s_{i}=0$ means that the source $s_{i}$ must not be trusted while $s_{i}=1$ means source $s_{i}$ is trusted.

Document Variables. $d_{i} \in\{0,1\}$ is, the random variable that represents the document objectivity. In that, $d_{i}=0$ means that the document is subjective whereas $d_{i}=1$ means that the document is objective.

175 Claim Variables. As mentioned above, each claim $c \in C$ is assigned a random variable, also denoted by $c \in\{0,1\}$, that indicates the correctness of the claim (1 denotes correctness).

Source Factors. As aforementioned, each source $s$ is associated with a normalized, multi-dimensional feature vector $\left\langle I_{1}^{S}(s), \ldots, I_{m_{S}}^{S}(s)\right\rangle$. The features can be derived from, e.g., engagement information (e.g. comments, likes), activity logs (e.g. number of documents posted), and demographic information (e.g. age, gender) $[13,24]$. Against this background, the source factor $f_{S}$ is defined as:

$$
f_{S}(s)=\exp \left(\sum_{i=1}^{m_{S}} w_{i}^{S} \times I_{i}^{S}(s) \times s\right)
$$

Here, $I_{i}^{S}(s) \in[0,1]$ is the feature score of the source for the $i$-th feature, which 
is normalized into the unit interval by dividing it by the maximum feature value observed among all sources. And $w_{i}^{S}$ is a weighting parameter indicating the significance of the individual features. Each source factor has a set of weight parameters $w^{S}=\left\{w_{1}^{S}, \ldots, w_{m_{S}}^{S}\right\}$.

Document factors. As aforementioned, each document $d$ is associated with a normalized, multi-dimensional feature vector $\left\langle I_{1}^{D}, \ldots, I_{m_{D}}^{D}\right\rangle$. We extract these features in the form of linguistic indicators such as discourse markers and affective phrases $[13,25]$. As a result, a document factor $f_{D}$ is defined as:

$$
f_{D}(d)=\exp \left(\sum_{i=1}^{m_{D}} w_{i}^{D} \times I_{i}^{D}(d) \times d\right)
$$

Here, $I_{i}^{D}(d) \in[0,1]$ is the feature score of the document for the $i$-th feature, which is normalized into the unit interval by dividing it by the maximum feature value observed among all documents. And $w_{i}^{D}$ is a weighting parameter indicating the significance of the individual features. Each document factor has a set of weight parameters $w^{D}=\left\{w_{1}^{D}, \ldots, w_{m_{D}}^{D}\right\}$.

Claims factors. Each claim factor $f_{C}$ depicts the causal relations between source, document, and claim.

$$
f_{C}(s, d, c)=\exp \left(w^{C} \times I(s, d, c)\right)
$$

where $w^{C}$ is the weight parameter of a given factor $f_{C}$ in the factor graph. Here, $I(s, d, c)$ is an indicator function capturing the consistency between source value, document value, and claim value as follows.

$$
I(s, d, c)= \begin{cases}1 & s=1 \wedge d=1 \wedge c=1 \\ & \text { or } s=0 \wedge d=0 \wedge c=0 \\ 0 \quad & s=1 \wedge(d \oplus c=0) \\ & \text { or } s=0 \wedge(d \oplus c=1) \\ 0.5 & \text { otherwise }\end{cases}
$$

This indicator captures several causal relations. First, a trustworthy source is likely to provide documents with credible claims and a non-trustworthy source is

likely to provide documents with non-credible claims. Second, a non-trustworthy 
source is unlikely to provide documents with credible claims and a trustworthy source is unlikely to provide documents with non-credible claims. For other cases, we assign a value of 0.5 to equally distribute the possibilities as default.

Handling opposing claims. Different Web documents may contain the same claim with opposite stances - support or refute it [26] - and a source is considered trustworthy, if it refutes an incorrect claim. A model that only eaptures that a claim was mentioned in a document by a source would neglect this aspect. However, incorporating such information explicitly via a new type of random variables (e.g. stance variables) would over-complicate the model, as their number would be larger than or equal to the number of documents, which is much larger than the number of claims (see our real-world datasets in $\S 7$ ). We therefore tackle this challenge by introducing an opposing variable $\neg c$ for each claim c. Then, model complexity increases only slightly: $C$ includes opposing claims, factor graph model contains a doubled number of parameters, and any document connects only to the positive or negative variable of a claim. However, as $c$ and $\neg c$ cannot have the same truth value, we enforce a non-equality constraint, as follows:

$$
\operatorname{Pr}\left(c, \neg c^{\prime}\right)= \begin{cases}0 & \text { if } c=c^{\prime} \\ \sum_{V} \operatorname{Pr}\left(c, \neg c^{\prime}, V \backslash\left\{c, c^{\prime}\right\}\right) & \text { otherwise. }\end{cases}
$$

Model Parameters. More precisely, we can define $W=\left\{w^{S}\right\}_{f_{S} \in F_{S}} \cup\left\{w^{D}\right\}_{f_{D} \in F_{D}} \cup$ $\left\{w^{C}\right\}_{f_{C} \in F_{C}} \cup\left\{w^{U}\right\}_{f_{U} \in F_{U}}$ as the set of weights for all factors. As a result, we can compute the representative probability distribution of the factor graph as the normalized product over all factors and variables:

$$
\begin{aligned}
\operatorname{Pr}(C, S, D ; W)= & \frac{1}{Z} \prod_{f_{S} \in F_{S}} \prod_{f_{D} \in F_{D}} \prod_{f_{C} \in F_{C}} f_{S}(s) \times f_{D}(d) \\
& \times f_{C}(s, d, c)=\frac{1}{Z} \prod_{f \in F} f(. ; W)=\frac{1}{Z} \exp \left(\sum_{w \in W} w \times I(.)\right)
\end{aligned}
$$

where $I($.$) is a feature or indicator function as aforementioned and Z=\sum_{C, S, D} \prod_{f \in F} f(. ; W)$

is a normalisation constant to ensure that the sum of probabilities over all configurations of $\{C, S, D, U\}$ is equal to one. Here the product $\prod_{f \in F}$ reflects the 
mutual relations, which are overlapping of causal relations (i.e. factors in factor graph). In other words, mutual relations leads to the factorization of causal relations to compute probability distributions.

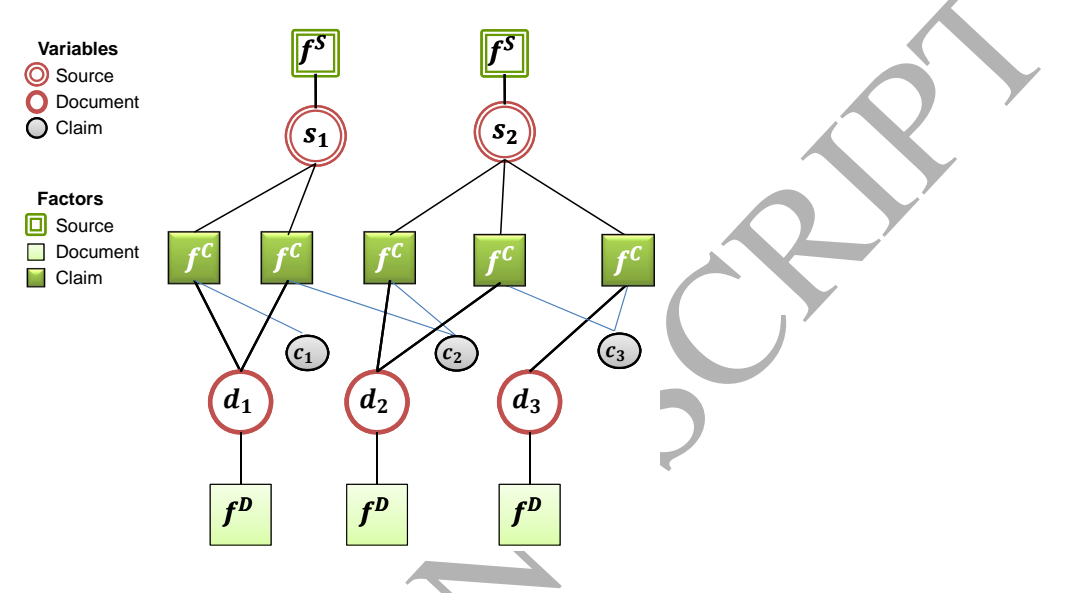

Figure 2: Credibility Extraction Model a respective factor graph. It comprises variables (shown as circles) for two sources $s_{1}, s_{2}$, three documents $d_{1}, d_{2}, d_{3}$ and three claims $c_{1}, c_{2}, c_{3}$. The figure also illustrates various factors, e.g., $f_{S}\left(s_{1}\right)$ is the source factor assigning a prior for the trustworthiness of source $s_{1}$ to the respective random variable. Likewise,

205 Factor $f_{C}\left(s_{1}, d_{1}, c_{1}\right)$ is a claim factor that connects source $s_{1}$, document $d_{1}$, and claim $c_{1}$, implying the causal relationship between them.

\subsection{Probability Computation}

The model of a factor graph enables us to compute the certainty of a claim in a factual networked data. This computation exploits the (marginal) probabilities of the random variables representing the correctness of claims. Following the model introduced above, claim variables are binary, so that $P(c=1$ ) (or $P(c)$ for short) is the probability that a claim $c \in C$ is correct. The computation of this probability is grounded in the correlations defined by the factor functions that relate the random variables to each other. 
Various techniques are available to compute probabilities in a factor graph, most commonly belief propagation or sampling [27]. The former considers the (un)certainty as information that is propagated through the factor graph and relies, for instance, on message-passing algorithms [28, 29]. Yet, it has been observed that belief propagation converges slowly if the graph is large and contains cycles [30]. When modeling networked data, the number of variables grows quickly and cyclic dependencies become the rule, rather than the exception. To cope with large and dense factor graphs, we resort to sampling to find the most probable values of random variables. Specifically, Gibbs sampling proved to be a highly efficient and effective mechanism for factor graphs [30]

Technically, we adopts the Expectation-Maximization (EM) principle [31] for probability computation on top of Gibbs sampling. The choice for EM is motivated by its generally fast convergence, computationally efficiency, and particular usefulness when the likelihood is an exponential function (i.e., maximising log-likelihood becomes maximising a linear function). In general, we compute the probability of the claims and produce Gibbs samples mutually. Given a model parameter $W$, the probability computation is iterative and alternates between an Expectation step (E-step) and a Maximization step (M-step), until convergence. Therefore, we obtain a sequence $\Omega^{0}, \Omega^{1}, \ldots, \Omega^{t}$ of Gibbs samples and a sequence $P^{0}, P^{1}, \ldots, P^{t}$ of credibility probabilities assigned to claims.

- E-step: We obtain a sequence of samples $\Omega^{t}$ by performing Gibbs sampling according to the probability distribution:

$$
q(C)=\operatorname{Pr}(C \mid D, S ; W) \propto \prod_{f \in F} P^{t-1}(c) f(. ; W)
$$

For handle opposing claims, we incorporate non-equality constraints (Eq. 6) into Gibbs sampling using an idea similar to [32], which, based on matrix factorisation, embeds constraints as factorised functions into the Markov chain Monte Carlo process. Note that $\Omega^{t}$ is a sequence, as any configuration of $C$ can appear multiple times. We weight the influence of causal interactions (i.e., factors) by the credibility of their contained claims, so 
that the credibility is propagated via mutual interactions (i.e., overlaps) between the factors.

- M-step: We estimate the credibility probabilities from the current Gibbs samples, for each claim $c$ :

$$
P^{t}(c)=\frac{\sum_{C \in \Omega^{t}} \mathbb{1}_{c=1}}{\left|\Omega^{t}\right|}
$$

The process converges when the difference between two consecutive estimates of credibility probabilities is insignificant.

245 3.3. Instantiation of credible facts

After computing probabilities from a given model parameter instance $W$, a deterministic assignment $g: C \rightarrow[0,1]$ may be instantiated anytime by predicting the truth values of all claims. Since claims are not independent, we take the truth configuration with their maximal joint probability:

$$
g(C)=\underset{C}{\arg \max } \operatorname{Pr}(C \mid D, S, U ; W)
$$

However, solving this equation is similar to solving a boolean satisfiability problem. Therefore, we may simply leverage the most recent Gibbs sampling result $\Omega$ obtained after the probability computation:

$$
g(C)=\underset{C}{\arg \max } \sum_{\omega \in \Omega} \mathbb{1}_{\omega=C}
$$

We break ties randomly. Since we are only interested in credible facts, we only retain a set of claims that we believe to be credible; i.e. $R=\{c \in C \mid$ $g(c)=1\}$. It is worth noting that the set of instantiated claims $R$ depends on the parameter instance $W$ and the factor graph model $M$, thus we denote $R=$ instantiate $(M, W)$.

Example 2. We are given a set of 3 unique claims $C=\left\{c_{1}, c_{2}, c_{3}\right\}$. For a given parameter instance $W$, the model has three Gibbs samples, $\omega_{1}=[1,1,0], \omega_{2}=$ $[1,0,0], \omega_{3}=[1,1,0]$, where the $i$-th element of the sample vector indicates the factual value ( 1 for credible and 0 for non-credible) of claim $c_{i}$. The instantiation 
will return $[1,1,0]$ as this vector appears most, which means its probability is maximal. That is, the model output of current parameter instance is $R=$ $\left\{c_{1}, c_{2}\right\}$.

This instantiation mechanism allows our model to gain a fine-grained control on the precision and the output size. In particular, it can automatically adjust the parameters to reduce the output size until the precision threshold is satisfied. For example, a perfect precision (1.0) can be achieved by only returning the output with a single correct claim $(|R|=1)$.

\section{Credibility Reinforcement with Precision Guarantee}

Problem 1 can be reformulated as a learning problem where we need to find a parameter instance $W$ from the multi-dimensional parameter space $[0,1]^{|W|}$ such that the precision of instantiated claims is guarantee (i.e. $\geq \tau$ ) and its output size is maximized. Formally, given a networked data $N=\langle S, D, C\rangle$, we can construct a factor graph model $M$ as above. Now given a precision threshold $\tau$, we need to find a parameter instance $W$ such that $\operatorname{Prec}(R) \geq \tau$ and $|R|$ is maximal, where $R=$ instantiate $(M, W)$.

For brevity sake, we denote $\operatorname{Prec}(W)=\operatorname{Prec}(R)$ as the precision of a given parameter instance, which is the fraction of actual credible claims over the total of instantiated claims. As the ground truth for all claims is unknown beforehand, we provide a precision estimation in Section 5. Similarly, we denote ${ }_{275} \operatorname{Size}(W)=|R|$ as the output size of a given parameter instance.

\subsection{Parameter Learning}

In general, to find the parameter instance that satisfies the constraint, it requires us to search over all the possible instances $[0,1]^{|W|}$, which is very large. In order to reduce the search space, we make two observations: 1) as there are 280 an infinite number of instances in the parameter space, an approximation is required to make it finite and 2) there is a tradeoff tendency between precision and output size that as we increase the feature weights of a parameter instance 

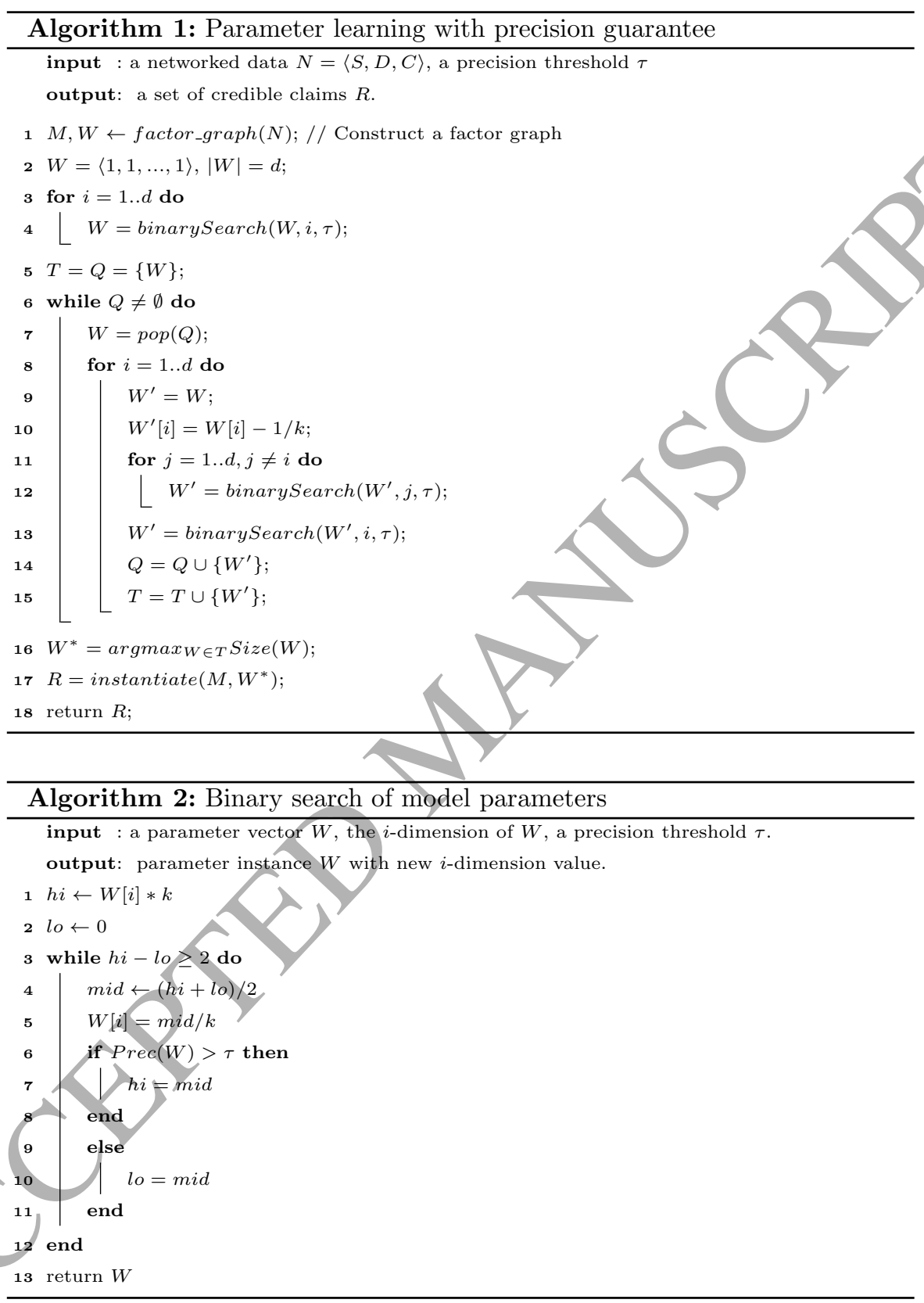

$I$, the precision increases while its output size decreases. The second observation comes from the fact that each weighting parameter reflects the perceived 

precision and maximizing output size: one can increase precision at the cost of output size. This property guarantees that the precision threshold can always be achieved. To achieve high precision, the model scarifies the recall by automatically learning a parameter instance that discards all uncertain outputs. For example, one can achieve a perfect precision (with high chance) by returning only the most credible claim (i.e. output size $=1$ ). 


\subsection{Learning Process}

The monotonicity assumption implies that there is a Pareto set [33] of pathreshold, which requires us to search through other dimensions $j \neq i \in[1, d]$ using binarySearch to make $W^{\prime}$ eligible for $T$ (line 11). However, we need to re-adjust the $i$-th dimension again to avoid oversatisfying the precision threshold (line 13). The algorithm halts when there is no such instance remaining (line 6). Then, we find the parameter instance with the largest output size (line 16) and from this parameter instance, we instantiate the set of credible claims (line 17). 
Example 3. Assume that we have three Pareto parameter instances $W_{1}=$ $(0.5,0.3), W_{2}=(0.6,0.2), W_{3}=(0.7,0.1)$ with the output size of $3,7,4$ re345 spectively. Alg. 1 initializes the parameter search with $W=(1,1)$. Using the binary search (line 11-line 13) from the first dimension to the last dimension, we will find $W_{1}, W_{2}$, and $W_{3}$ in this order. Finally, we compare the output size among them and choose $W_{2}$, which both satisfies the precision threshold and has a maximal output size.

\section{Precision Estimation}

We now discuss the implementation of the precision éstimation (i.e. $\operatorname{Prec}(W)$ call) in Alg. 2. Since the number of claims is large, it would be costly to obtain ground truth for all claims. Instead, it is practical to ask ground truth for a number of sample claims (e.g. available training data or expert annotations).

355 In addition, asking for labels for many claims goes against the purpose of our setting, which is finding a set of credible claims with little or only existing effort.

\subsection{Labeling effort minimization}

To this end, we approximate the precision of the instantiated claims $R$ using sampling technique. There is a trade-off between the confidence of precision estimation and the labeling effort (i.e. the number of oracle probes), see Fig. 3. Intuitively, if all claims can be labeled, the precision estimation is more confident, and the recall of instantiation is also better. However, the labeling effort is limited and thus our goal is to design different strategies to minimize the number of oracle probes without sacrificing the confidence level of precision estimation.

To this end, we propose a guided sampling technique as follows. To estimate the precision of $R=$ instantiate $(M, W)$, we do three steps:

1. First, we create a sample $C^{\prime} \subset R$ using Monte-Carlo process [32]. That is, the sampler is guided by the probability distribution in Eq. 7. In other words, each claim has a sampling probability proportional to its marginal probability (Eq. 9). In the end, the majority of the samples will include 


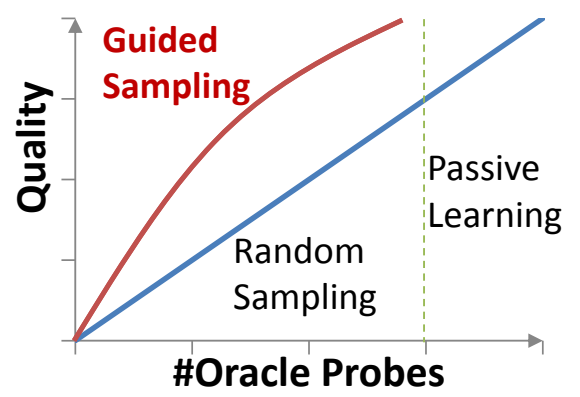

Figure 3: Different strategies of precision estimation

probably credible claims since we want to maximíze the recall. Moreover, this guidance helps the samples reflect the true distribution of data and make the precision estimation more accurate [34]. In practice, we fix $\left|C^{\prime}\right|=10 \log (|R|)$ to make the sample size adaptively to different output. The choice of $\log$ function is due to the long-tail property of Web data [35] (i.e. a few claims are far important than the large number of other claims).

2. We ask an oracle to label the selected claims. The result is called $h\left(C^{\prime}\right)$.

3. We compute the precision of $C^{\prime}$ against $h\left(C^{\prime}\right)$ (i.e. $\sum_{c \in C^{\prime}} \mathbb{1}_{h(c)=1} /\left|C^{\prime}\right|$ ), which is the estimated precision of $R$.

We further discuss two other sampling strategies as baselines:

- Random sampling: this strategy chooses randomly a set of claims for labeling. And the precision is estimated by applying the current model parameters on the sampled data.

- Passive learning: this strategy is only applicable for static data, where a limited list of labeled claims is provided before-hand. Every time the model needs to estimate the precision, it will test its current parameters on the labeled data to see how many of them are correctly labeled and get the estimated precision value. If the labeled data is big, we could get a good estimation with the trade-off that the effort to obtain it is 


\section{Reusing samples. Although the above approach only requires a small number} of samples per call, the total number of samples across different calls to estimate precision might be high since the selected samples can be different. To this end, we introduce a technique to reuse the samples from different calls to reduce the total cost to obtain the samples. That is, we order the claims in $C$ based on a hash value of the claims. As a result, in order to sample claims from $R$, we pick the claims by the increasing order of hash values. This increases the chance of reusing claims in different calls to the oracle, hence, reducing the cost to obtain the labels for the claims.

${ }_{410}$ The hash function is designed in such a way that the probability distribution of data is preserved [36]. To do this, we compute the importance of each claim as the number of data sources and the number of their documents sharing this claim. Intuitively, the importance distribution of claims aligns with their probability distribution, since credible/non-credible claims are often given by similar number of trustworthy/untrustworthy sources. As a result, the order of hash values is the order of importance degrees of claims.

Reducing number of precision estimations. To minimize the labeling cost (i.e. the oracle probes), we will further reduce the number of precision estimations (i.e. $\operatorname{Prec}(W)$ calls). The core idea is that to exploit the aforementioned 

(§5.1) might actually increase the number of precision estimations. To avoid this problem, we leverage the monotonicity and anti-monotonicity properties together as follows. Given an instance $W$ in the anti-Pareto set $T^{\prime}$, if there does not exist any $W^{\prime}$ in the Pareto set $T$ such that $W \prec W^{\prime}$, then $\operatorname{Prec}(W) \geq \tau$.

${ }_{455}$ This is because Pareto and anti-Pareto sets contain instances that are minimally valid $(\operatorname{Prec}(W) \geq \tau)$ and maximally invalid $(\operatorname{Prec}(W)<\tau)[33]$. 


\section{Credibility Reinforcement with Noisy Data}

Some applications may contain noise that causes occasional violation of the monotonic rule. Even though this reasonable rule is expected to hold on most pairs of parameters, it is quite over-optimistic to assume that it would hold on all pairs.

We propose a noisy parameter learning that works in an interactive manner. In this approach, the system asks the oracle to evaluate precision (i.e., $\operatorname{Prec}(W)$ call) and uses the monotone rule to find the best parameter that satisfies the precision threshold with maximum output size, taking into aecount the fact that the monotone rule may sometimes be violated (e.g. expert proyides the wrong label by mistake in the aforementioned guided sampling procedure). Accordingly, now there are two objectives. First, a good algorithm should ask to probe (a.k.a. label) as few claims as possible. Second, due to the existence of noise, the algorithm should also minimize the number of claims mis-instantiated.

Technically, we cater for erroneous labels by augmenting the precision estimation with a lightweight confirmation check. This check is triggered after a fixed number of iterations of the parameter learning process and proceeds as follows. For every claim $c$ for which label $h(c)$ has been provided, a deterministic assignment $g_{\sim c}$ is constructed based on the current parameter from which the label for $c$ has been excluded. This is efficiently implemented by copying current model parameters and running the probability computation and instantiation with $c$ as unlabeled claim. Then, the instantiation for claim $c$ in $g_{\sim_{c}}$ is compared with the respective label $h(c)$. If $g_{\sim c}(c) \neq h(c)$, then $h(c)$ is identified

470 as an erroneous label. Upon each detected noise, we allow the expert to fix the mistake and increment the labeling effort by 1 .

The intuition is as follows. At the early iteration $i$ of parameter learning, the deterministic assignment $g^{i}$ is immature and gives an incorrect instantiation for claim $c$ and the label of precision estimation is also incorrect. However, when we 475 run the check for erroneous labels, it happens at a later iteration $j$. As a result, the deterministic assignment constructed at this point $g^{j}$ is mature, thus it is 
different from the deterministic assignment at the early iteration. Therefore, the true value for claim $c$ assigned by $g_{\sim c}^{j}$ is different from one assigned by $g^{i}$ and as $g_{\sim c}^{j}$ is constructed with more labels and better model parameters, they may give a better value for claim $c$.

\section{Experimental Evaluation}

In this section, we evaluate the proposed approach experimentally, using realworld datasets. We first discuss the experimental setup (\$7.1), before turning to an evaluation of the following aspects of our approach:

- The indicative power of credibility features $(\S 7.2)$.

- The runtime performance of credibility extraction (\$7.3).

- The correctness of learning model (\$7.4).

- The effectiveness of learning model ( $\$ 7.5)$.

- The robustness against noisy data (\$7.6).

Since different baselines are not applicable for all experimental setting, we defer the description of baselines to each evaluation.

\subsection{Settings}

Datasets. We utilize the following datasets (see also Table 1):

- Health: The dataset contains 291276 claims about side-effects of drugs extracted from $2.8 \mathrm{M}$ documents of $15 \mathrm{~K}$ users on a healthcare forum (healthboards.com) [37]. We consider 72819 claims that have been labelled by health experts. The claims are part of 661882 documents from 11206 users/sources.

- Snopes: This dataset contains a wide range of claims from news websites, social media, e-mails, etc [18]. The credibility of these claims has been assessed by Snopes' editors. The dataset comprises 80421 documents of 23260 sources that contain 4856 labelled claims. 
- Wikipedia: This dataset contains a list of proven hoaxes and fictitious people from Wikipedia links [38]. In total, there are 1955 sources, 3228 documents, and 157 labelled claims.

Table 1: Datasets

\begin{tabular}{llll}
\hline Dataset & \#Sources & \#Documents & \#LabeledClaims \\
\hline health & 11206 & 661882 & 72819 \\
snopes & 23260 & 80421 & 4856 \\
wiki & 1955 & 3228 & 157 \\
\hline
\end{tabular}

For those datasets, we derive credibility features as follows. If a source is a website, we rely on ranking scores [39]. If a source is an author, features include personal information (age, gender) and activitylogs (number of documents) [24]. Credibility of documents is assessed using common linguistic features such as stylistic indicators (e.g. usage of modals, named entities, inferential conjunction) and affective indicators (e.g. sentiments, thematic words) [40]. In our experiments, we use the ground truth of the datasets to simulate oracle probes.

Evaluation Metrics. We relied on the following measures:

Relative label efforts $\left(E_{i}\right)$ is the number of oracle calls $i$ relative to the number 515 of claims $|C|$ in the dataset, i.e., $E=i /|C|$.

Actual precision (Prec) measures the correctness of the returned set $R$ of credible claims:$$
\hat{\operatorname{Prec}}=\frac{\left|\left\{c \in R \mid g(c)=g^{*}(c)\right\}\right|}{|R|}
$$

where $g^{*}: C \rightarrow\{0,1\}$ is the correct assignment of truth values for all claims (i.e. ground truth).

Actual recall $(\hat{\operatorname{Rec}})$ : since the output is a maximal set of credible claims with precision guarantee, the actual recall is the ratio of output size over all credible claims:

$$
\hat{\operatorname{Re}}=\frac{|R|}{\left|\left\{c \in C \mid g^{*}(c)=1\right\}\right|}
$$
}


Experimental environment. Experimental results have been obtained on an Intel Core i7 system (3.4GHz, 12GB RAM). The number of Gibbs samples ${ }_{520}$ is fixed to $1 \mathrm{M}$. The experimental results are averaged over 10 runs to avoid randomness.

\subsection{Indicative power of credibility features}

We first carried out an experiment to verify the monotonicity assumption. For each claim, we compute their features. Then, for different values of these

525 features, we report the precision of the claims, by computing the fraction of credible claims among the claims whose feature values fallen into the given range.
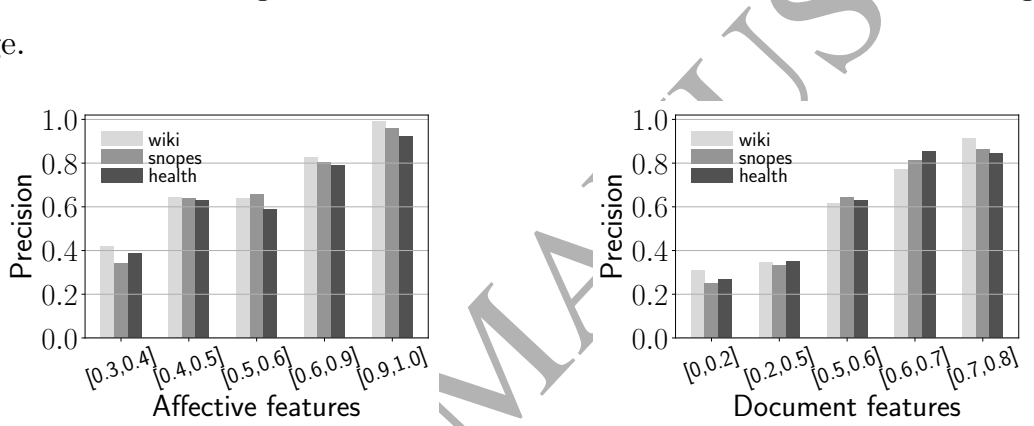

Figure 4: Indicative power of credibility features

Figure 4 shows the monotonicity result for affective features and document length features. The $\mathrm{X}$-axis is the value of the features. The Y-axis presents the "precision" of the respective claims. The key finding is that as the values of the affective features increase, the precision of corresponding claims is nondecreasing. For example, when the values of the affective features increases from $[0.6,0.9]$ to $[0.9,1.0]$, the precision increases from 0.81 to 0.95 in average. We observe the same trend for the document length features, even though the monotonicity only holds from 0 to 0.8 in average. This shows that our monotonicity assumption is valid in real datasets. 


\subsection{Runtime Performance}

This experiment measures the total running time of Alg. 1 for finding the best model parameter with maximal instantiation and precision guarantee; i.e. returning a set of credible claims given an unlabeled data. We evaluate our precision estimation method across different datasets with different baselines: guided - the proposed approach in $\S 5$, random - random sampling, and passive - the passive learning setting where the same amount of labels is provided before-hand (but their distribution is unknown).
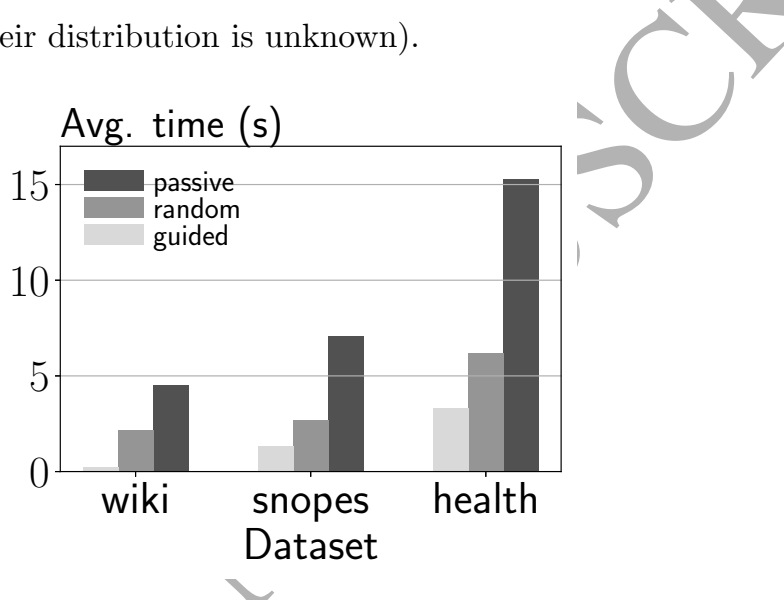

Figure 5: Runtime performance

Fig. 5 shows the obseryed running time. With large dataset size (wiki to health), the response time increases. The guided method is the fastest since it provides accurate precision estimation, helping model parameters converge quickly. However, it is worth noting that we use ground truth to simulate oracle probes. Capturing interaction time with oracle requires further cost models, which is out of the scope of our paper.

\subsection{Correctness of learning model}

In this experiment, we study the correctness of our learning model in finding credible claims. The idea is that the more labels are elicited from oracle, the better the model should be in returning credible claims. Regarding the setting, 555 we set the precision threshold $\tau=0.9$ and modify the Alg. 1 such that the 
trusted set of claims is instantiated for each oracle probe (i.e. precision estimation). We compare our precision estimation with the baselines: (i) guided the proposed sampling in $\S 5$ and (ii) random - random sampling. The passive learning method is not applicable in our setting and can be interpreted as the random baseline with varying amount of labels whose distribution is arbitrary.

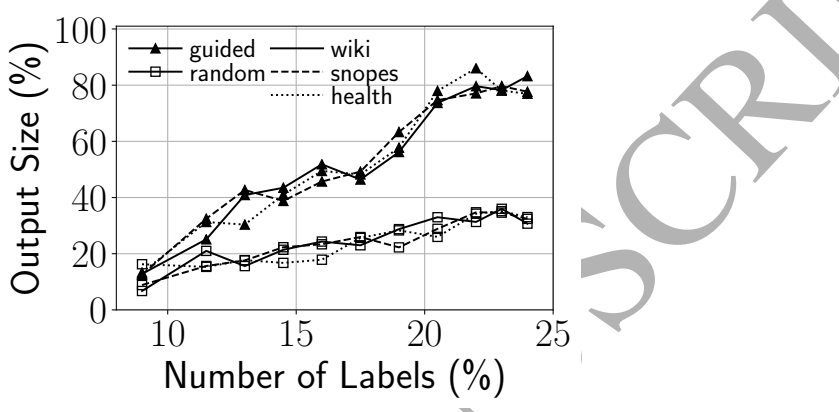

Figure 6: Correctness of learning model

Figure 6 depicts the results. The $\mathrm{X}$-axis presents the percentage of labels used by the process (accumulated after each precision estimation call). The Y-axis presents the corresponding output size. It can be clearly seen that the more labels are provided, the output size of instantiated claims increases in average. This represents the effectiveness of our learning process in using the labels as we can instantiate most of the credible claims without too much efforts. Moreover, our guided sampling outperforms random sampling, which indicates the correctness of the computed probability information.

Deep understandings of the learning process. We further provide the characteristics of our learning model given different precision requirements. In particular, we vary the precision threshold from $\tau=0.8$ to $\tau=0.9$. Then we report the evaluation metrics of learning process, including the label effort, the actual precision, and the actual recall. Regarding the setting, we perform the experiment on the claims whose labels are known as we want to compute the 575 actual precision by comparing the instantiated claims with the oracle labels.

Table 2 illustrates the result (we show only the average across datasets for 
Table 2: Characteristics of learning process

\begin{tabular}{lrrr}
\hline \multirow{2}{*}{ Metric } & \multicolumn{2}{c}{$\tau$ : precision threshold } \\
\cline { 2 - 4 } & 0.8 & 0.85 & 0.9 \\
\hline Label effort & $21 \%$ & $28 \%$ & $24 \%$ \\
Precision & 0.82 & 0.84 & 0.91 \\
Recall & 0.78 & 0.75 & 0.72 \\
\hline
\end{tabular}

brevity sake). An important observation is that as we increase the/precision threshold, the recall decreases, which is the traditional trade-off between maximizing precision and maximizing recall. However our approach allows the explicit control of precision. Another noticeable observation is that the actual precision always satisfies the precision threshold. Although at $\tau=0.85$, the actual precision is 0.84 but the difference is negligible. The final finding is that our learning process only require a small portion of labeled claims in order to achieve the quality requirement.

\subsection{Effectiveness of learning model}

In this experiment, we compare the effectiveness of our algorithm with the baseline w.r.t. the output size of instantiated claims. Existing classification techniques (e.g. J48 decision tree [41], SVM-RBF [42], DT-Rank [43]) do not support precision guarantee (i.e. their result has an arbitrary precision). Since ground truth is not known in advance, we derive a baseline applicable for our problem. The baseline method constructs a classifier $L$ of the form $L(c)=\sum_{i} w_{i} I_{i}$ where $w_{i}$ are real numbers and each claim $c$ has the same features $\left\langle I_{1}, I_{2}, \ldots\right\rangle$ as our method. In other words, the classifier is a weighted combination of the features, where each weight describes the importance of the corresponding feature. In order to learn the weights, we leverage the same labels used by our method and update the parameters by the MIRA algorithm[44]. In order to guarantee the precision, we set a threshold $\delta$ for the classifier to select the credible claims. For a claim $c$, if $L(c)>\delta$, claim $c$ is considered credible and 
vice versa. The threshold $\delta$ allows us to control the precision of the returned

claims by the baseline method. The higher the threshold, the higher the precision. After constructing the classifier, we use binary search to find a smallest possible threshold $\delta$ such that the returned claims have a precision higher than the precision constraint $\tau$.

Figure 7 presents the result. The $\mathrm{X}$-axis varies the precision threshold from $\tau=0.8$ to $\tau=0.96$. The Y-axis is the ratio of output size between our learning model and the baseline. We observe that our algorithm outperforms the baseline significantly. For instance, when the precision constraint is 0.82 , we are able to return 6 times the amount of the baseline in average. The above experiments show that our approach is not only able to satisfy the precision guarantee but also with higher output size. This can be explained as follows. When the precision threshold is low, the baseline often over-satisfies the requirement and thus instantiate far less number of claims than our model. When the precision threshold is high, the over-satisfying becomes negligible and thus the baseline performs better than before. However, it is still worse than our model since it 615 does not taking into account the relationships between sources, documents, and claims as captured by our probabilistic model.

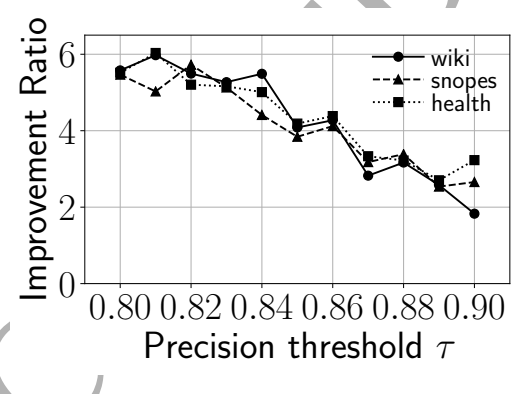

Figure 7: Effectiveness of learning model

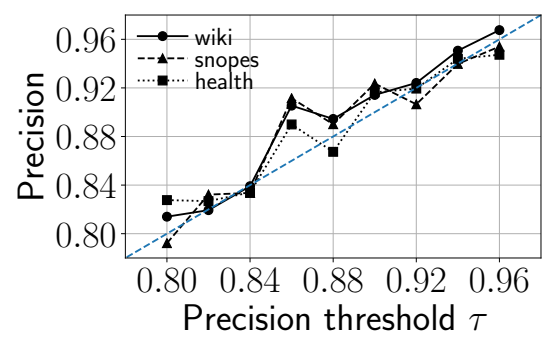

Figure 8: Effectiveness of precision estimation

Effectiveness of precision estimation. The core of our learning model is the precision estimation to guide the search of parameters. In this setting, we would like to verify the accuracy of our precision estimation using guided sampling as 
proposed in $\S 5$. To this end, we will compare the precision of the output with the precision requirement.

Fig. 8 illustrates the effectiveness of our precision estimation. The experiment is conducted by varying precision threshold from $\tau=0.80$ to $\tau=0.96$. Our algorithm is able to return the claims that satisfy the precision constraint. This can be seen as the precision values of our algorithm are near the identity line. For instance, when the precision constraint is 0.9 , the instantiated claims have a precision of 0.92 in average. In some cases, the precision is less than the threshold but the difference is negligible.

\subsection{Robustness against Noisy Data}

Detecting noisy data. We evaluate our approach to detect erroneous input by simulating oracle mistakes. With a given probability $p$, we transform correct label input into an incorrect label. In other words, the oracle labels with confidence level of $\alpha=1-p$ (varying from $80 \%$ to $95 \%$ ). We consider all datasets and trigger the confirmation check (see \$6) after each 10 iterations. Table 3 shows the percentage of detected noises when decreasing parameter $\alpha$. Across all datasets, the majority of inserted noises is detected.

Table 3: Detected noises (\%)

\begin{tabular}{ccccc}
\hline \multirow{2}{*}{ Dataset } & \multicolumn{4}{c}{$\alpha$ : confidence level } \\
\cline { 2 - 5 } & $95 \%$ & $90 \%$ & $85 \%$ & $80 \%$ \\
\hline wiki & 100 & 100 & 97 & 88 \\
snopes & 100 & 100 & 93 & 85 \\
health & 100 & 96 & 86 & 78 \\
\hline
\end{tabular}

\section{)}

Learning effectiveness with noisy data. We now show that the noisy learning process is still effective in terms of label effort, precision, and recall. For the setting, we fix the precision threshold $\tau=0.9$ and vary the confidence level $\alpha$ from $80 \%$ to $95 \%$. The experimental result is presented in Table 4 (we show only the average across datasets for brevity sake). The key finding is that with a 
reasonable level of noise, e.g. $\alpha=90 \%$, our approach still satisfies the precision threshold.

Table 4: Learning effectiveness with noisy data

\begin{tabular}{lllll}
\hline \multirow{2}{*}{ Metric } & \multicolumn{4}{c}{$\alpha$ : confidence level } \\
\cline { 2 - 5 } & $80 \%$ & $85 \%$ & $90 \%$ & $95 \%$ \\
\hline Label effort & $31 \%$ & $28 \%$ & $25 \%$ & $24 \%$ \\
Precision & 0.85 & 0.88 & 0.90 & 0.91 \\
Recall & 0.63 & 0.67 & 0.70 & 0.72 \\
\hline
\end{tabular}

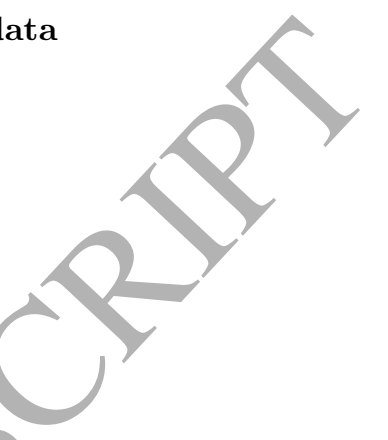

We conclude with a remark that, the learning performance relies on noisy data being small. This is reasonable because, intuitively, when the amount of noise is large, it would be very difficult to recover most labels correctly without checking the entire dataset.

\section{Related Work}

Active learning. Active learning approaches allows the learning algorithm to choose the data it wants to get labels, which is suitable in settings where the labels are costly to obtain. It has been applied in various machine learning applications such as information fusion [45, 46], speech recognition, information extraction [47] and text classification [48]. In credibility assessment, active learning approaches can be classified into two categories: classifier-independent 655 and classifier-specific. Classifier-specific approaches requires constructing a classifier. Notable works in this category are SVMs [48] and decision trees [49] while committee-based approaches [47] belong to classifier-independent category. Although our approach is similar to active learning approaches, there is a fundamental difference. Traditional active learning approaches do not guarantee that the values returned by the learning algorithm satisfy a predefined quality. Moreover, they still require a lot of training data, which is costly, and might 
not reach the required quality [50]. On the other hand, our approach is able to achieve this constraint while maximizing the output size.

Knowledge Extraction. Given a set of data items claimed by multiple sources, the knowledge extraction (a.k.a. truth finding) problem is to determine the true values of each claim $[51,52]$, with various usages in information corroboration, data fusion, and data collection [53, 54, 55, 56, 57]. Existing work on knowledge extraction also models the mutual reinforcing relationship between data items and sources, e.g., by a Bayesian model, maximum likelihood estimation, and latent credibility analysis $[58,59,60,61,62]$. In addition, these techniques often incorporate prior knowledge about various aspects of the source and the data, such as the dependence between sources [63] and the temporal dimension in evolving data [64]. Although these techniques are able to estimate the truthfulness of the claims, they do not have a precision guarantee on the truth estimation.

Credibility Features. A lot of prior research have been conducted on identifying credible claims from the Web $[65,10,66,67,68]$. In these works, they capture the credibility of a claim as a combination of individual features/indicators. The first type of features is content-based, such as semantic features (e.g. category, entities, keywords), sentiments features (e.g. subjectivity), and syntactic features (part-of-speech tag, punctuation marks, spelling errors), advertisements, and page layout $[69,70,71,72]$. The second type of features is network-based, such as the overall ratings of sources sharing the same claims. However, most of the existing works only compute the credibility as an aggregation function of these features; and again, do not provide a precision guarantee. On top of these works, we reuse these features and additionally take into account the mutual relationships between sources, their documents, and claims by the factor graph model to support the precision guarantee process. 


\section{Conclusions and Future Work}

[2] L. B. S. Network, Big data fusion in internet of things, Information Fusion 40 (2017) 32-33.

[3] F. Wu, Y. Huang, Z. Yuan, Domain-specific sentiment classification via fusing sentiment knowledge from multiple sources, Information Fusion 35 715

This paper proposed techniques to find credible claims in Web contents with a precision guarantee. The approach comprises of two components: a probabilistic model and a learning process. The former is responsible for capturing the mutual reinforcing relationships between sources, documents, and claims and enforce the consistency observations about credibility. For example, a trustworthy source is likely to provide credible claims and vice-versa. The instantiation of the probabilistic model is a set of (probably) credible claims, which is the output. The latter searches for the best-suitable parameters of the probabilistic model such that the output precision satisfying a pre-defined threshold and the output size is maximal. Our evaluation showed that our techniques outperform respective baselines significantly, up to 6 times better.

In future work, as online Web sources are often divided by communities with a wide range of characteristics, we aim to extend the probabilistic model to capture the community membership of sources. In addition, we also intend to extend our approach to handle continuous/multi-set labels as the current 705 approach only supports discrete labels.

\section{References}

\section{References}

[1] D. E. Losada, J. Parapar, A. Barreiro, A rank fusion approach based on score distributions for prioritizing relevance assessments in information retrieval evaluation, Information Fusion 39 (2018) 56-71.

$$
\text { (2017) 26-37. }
$$


[4] O. Deshpande, D. S. Lamba, M. Tourn, S. Das, S. Subramaniam, A. Rajaraman, V. Harinarayan, A. Doan, Building, maintaining, and using knowledge bases: A report from the trenches, in: SIGMOD, 2013, pp. 1209-1220.

[5] M. T. Dzindolet, S. A. Peterson, R. A. Pomranky, L. G. Pierce, H. P. Beck,

[12] M. Banko, M. J. Cafarella, S. Soderland, M. Broadhead, O. Etzioni, Open information extraction for the web, in: IJCAI, 2007, pp. 2670-2676.

\section{(1)}

[13] S. Mukherjee, G. Weikum, C. Danescu-Niculescu-Mizil, People on drugs: Credibility of user statements in health communities, in: KDD, 2014, pp. 65-74.

${ }_{740}[14]$ A. K. Raz, C. R. Kenley, D. A. DeLaurentis, A system-of-systems perspective for information fusion system design and evaluation, Information Fusion 35 (2017) 148-165. 
[15] C. De Maio, G. Fenza, V. Loia, M. Parente, Time aware knowledge extraction for microblog summarization on twitter, Information Fusion 28 (2016) $745 \quad 60-74$.

[16] L. Snidaro, J. García, J. Llinas, Context-based information fusion: a survey and discussion, Information Fusion 25 (2015) 16-31.

[17] P. Ernst, C. Meng, A. Siu, G. Weikum, Knowlife: a knowledge graph for health and life sciences, in: ICDE, 2014, pp. 1254-1257.

[18] Snopes, http://resources.mpi-inf .mpg.de/impact/web_ credibility_analysis/Snopes.tar.gz (2017).

[19] S. S. Saquib, C. A. Bouman, K. Sauer, Ml parameter estimation for markov random fields with applications to bayesian tomography, IEEE Transactions on Image Processing 7 (7) (1998) 1029-1044.

${ }_{755}[20]$ N. Q. V. Hung, C. T. Duong, N. T. Tam, M. Weidlich, K. Aberer, H. Yin, X. Zhou, Argument discoyery via crowdsourcing, VLDBJ 26 (4) (2017) $511-535$.

[21] N. Q. Hung, D. C. Thang, N. T. Tam, M. Weidlich, K. Aberer, H. Yin, $\mathrm{X}$. Zhou, Answer validation for generic crowdsourcing tasks with minimal efforts, VLDBJ 26 (6) (2017) 855-880.

[22] N. Q. V. Hung, D. C. Thang, M. Weidlich, K. Aberer, Minimizing efforts in validating crowd answers, in: SIGMOD, 2015, pp. 999-1014.

[23] N. Q. V. Hung, H. H. Viet, N. T. Tam, M. Weidlich, H. Yin, X. Zhou, Computing crowd consensus with partial agreement, TKDE 30 (1) (2018) $1-14$.

[24] C. Yu, B. Xiao, D. Yao, X. Ding, H. Jin, Using check-in features to partition locations for individual users in location based social network, Information Fusion (2017) 86-97. 
[25] A. Tommasel, D. Godoy, A social-aware online short-text feature selection technique for social media, Information Fusion (2018) 1-17.

[26] K. S. Hasan, V. Ng, Why are you taking this stance? identifying and classifying reasons in ideological debates., in: EMNLP, 2014, pp. 751-762.

[27] S. Peng, A. Yang, L. Cao, S. Yu, D. Xie, Social influence modeling using information theory in mobile social networks, Information Sciences 379 (2017) 146-159.

[28] F. R. Kschischang, B. J. Frey, H.-A. Loeliger, Factor graphs and the sumproduct algorithm, TIT (2001) 498-519.

[29] A. Abrardo, M. Barni, K. Kallas, B. Tondi, A message passing approach for decision fusion in adversarial multi-sensor networks, Information Fusion (2018) 101-111.

[30] C. Zhang, C. R, Towards high-throughput gibbs sampling at scale: A study across storage managers, in: SIGMOD, 2013, pp. 397-408.

[31] A. McCallum, K. Bellare, F. Pereira, A conditional random field for discriminatively-trained finite-state string edit distance, in: UAI, 2005, pp. 388-395.

[32] M. Schmidt, Linearly constrained bayesian matrix factorization for blind source separation, in: NIPS, 2009, pp. 1624-1632.

[33] S. Borzsony, D. Kossmann, K. Stocker, The skyline operator, in: ICDE, 2001, pp. 421-430.

${ }_{790}[34]$ M. Bach, A. Werner, J. Żywiec, W. Pluskiewicz, The study of under-and over-sampling methods utility in analysis of highly imbalanced data on osteoporosis, Information Sciences 384 (2017) 174-190.

[35] H. Kwak, C. Lee, H. Park, S. Moon, What is twitter, a social network or a news media?, in: WWW, 2010, pp. 591-600. 
[36] O. Amble, D. E. Knuth, Ordered hash tables, The Computer Journal (1974) 135-142.

[37] Healthboards, http://resources.mpi-inf .mpg.de/impact/ peopleondrugs/data.tar.gz (2017).

[38] Wikipedia, http://resources.mpi-inf.mpg.de/impact/web credibility_analysis/Wikipedia.tar.gz (2017).

[39] C. Ding, X. He, P. Husbands, H. Zha, H. Simon, Pagerank, hits and a unified framework for link analysis, in: SDM, 2003, pp. 249-253.

[40] A. Olteanu, S. Peshterliev, X. Liu, K. Aberer, Web credibility: Features exploration and credibility prediction, in: ECIR, 2013, pp. 557-568.

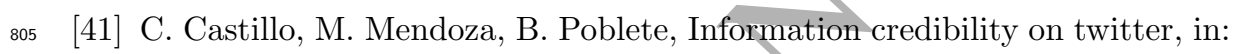
WWW, 2011, pp. 675-684.

[42] F. Yang, Y. Liu, X. Yu, M. Yang, Automatic detection of rumor on sina weibo, in: KDD, 2012, p. 13.

[43] Z. Zhao, P. Resnick, Q. Mei, Enquiring minds: Early detection of rumors in social media from enquiry posts, in: WWW, 2015, pp. 1395-1405.

[44] K. Crammer, O. Dekel, J. Keshet, S. Shalev-Shwartz, Y. Singer, Online passive-aggressive algorithms, JMLR (2006) 551-585.

[45] P. Pérez-Gállego, J. R. Quevedo, J. J. del Coz, Using ensembles for problems with characterizable changes in data distribution: A case study on quantification, Information Fusion 34 (2017) 87-100.

[46] B. Pes, N. Dessì, M. Angioni, Exploiting the ensemble paradigm for stable feature selection: A case study on high-dimensional genomic data, Information Fusion 35 (2017) 132-147.

[47] S. Argamon-Engelson, I. Dagan, Committee-based sample selection for probabilistic classifiers, JAIR (1999) 335-360. 
[48] S. Tong, D. Koller, Support vector machine active learning with applications to text classification, JMLR (2002) 45-66.

[49] B. Zadrozny, C. Elkan, Learning and making decisions when costs and probabilities are both unknown, in: KDD, 2001, pp. 204-213.

${ }_{825}$ [50] B. Settles, Active learning, Synthesis Lectures on Artificial Intelligence and Machine Learning 6 (1) (2012) 1-114.

[51] R. R. Yager, F. Petry, An intelligent quality-based approach to fusing multisource probabilistic information, Information Fusion (2016) $127-136$.

[52] W. Xu, J. Yu, A novel approach to information fusion in multi-source datasets: A granular computing viewpoint, Information Sciences (2017) $410-423$.

[53] A. Galland, S. Abiteboul, A. Marian, P. Senellart, Corroborating information from disagreeing views, in: WSDM, 2010, pp. 131-140.

[54] X. L. Dong, F. Naumann, Data fusion: resolving data conflicts for integration, in: VLDB, 2009, pp. 1654-1655.

[55] J. A. Balazs, J. D. Velásquez, Opinion mining and information fusion: a survey, Information Fusion (2016) 95-110.

[56] J. Zhang, V. S. Sheng, Q. Li, J. Wu, X. Wu, Consensus algorithms for biased labeling in crowdsourcing, Information Sciences (2017) 254-273.

[57] N. Q. Y. Hung, N. T. Tam, M. Weidlich, D. C. Thang, X. Zhou, What-if analysis with conflicting goals: Recommending data ranges for exploration, in: VLDB, Vol. 10, 2017.

[58] B. Zhao, B. I. Rubinstein, J. Gemmell, J. Han, A bayesian approach to discovering truth from conflicting sources for data integration, in: VLDB, 2012, pp. 550-561. 
[59] D. Wang, L. Kaplan, H. Le, T. Abdelzaher, On truth discovery in social sensing: A maximum likelihood estimation approach, in: IPSN, 2012, pp. $233-244$.

[60] J. Pasternack, D. Roth, Latent credibility analysis, in: WWW, 2013, pp. 850 1009-1020.

[61] A. Kozierkiewicz-Hetmańska, The analysis of expert opinions consensus quality, Information Fusion (2017) 80-86.

[62] A. Šipošová, L. Šipeky, F. Rindone, S. Greco, R. Mesiar, Super-and subadditive constructions of aggregation functions, Information Fusion 34 (2017) 49-54.

[63] X. L. Dong, L. Berti-Equille, Y. Hu, D. Srivastava, Solomon: Seeking the truth via copying detection, in: VLDB, 2010, pp. 1617-1620.

[64] X. L. Dong, L. Berti-Equille, D. Srivastava, Truth discovery and copying detection in a dynamic world, in: VLDB, 2009, pp. 562-573.

[65] T. G. Papaioannou, J.-E. Ranvier, A. Olteanu, K. Aberer, A decentralized recommender system for effective web credibility assessment, in: CIKM, 2012 , pp. $704-713$.

[66] H. Yin, H. Chen, X. Sun, H. Wang, Y. Wang, Q. V. H. Nguyen, Sptf: a scalable probabilistic tensor factorization model for semantic-aware behavior prediction, in: ICDM, 2017, pp. 585-594.

[67] N. T, Tam, M. Weidlich, D. C. Thang, H. Yin, N. Q. V. Hung, Retaining data from streams of social platforms with minimal regret, in: IJCAI, 2017, pp. $2850-2856$.

[68] H. Chen, H. Yin, W. Wang, H. Wang, Q. V. H. Nguyen, X. Li, Pme: Projected metric embedding on heterogeneous networks for link prediction, in: KDD, 2018, pp. 1177-1186. 
[69] A. Valdivia, M. V. Luzón, E. Cambria, F. Herrera, Consensus vote models for detecting and filtering neutrality in sentiment analysis, Information Fusion 44 (2018) 126-135.

${ }_{875}^{6}$ [70] I. Chaturvedi, E. Cambria, R. E. Welsch, F. Herrera, Distinguishing between facts and opinions for sentiment analysis: Survey and challenges, Information Fusion 44 (2018) $65-77$.

[71] S. Poria, E. Cambria, R. Bajpai, A. Hussain, A review of affective computing: From unimodal analysis to multimodal fusion, Information Fusion 37 (2017) 98-125.

[72] E. Cambria, Affective computing and sentiment analysis, IEEE Intelligent Systems 31 (2) (2016) 102-107. 
Maximal Fusion of Facts on the Web with Credibility Guarantee

Nguyen Thanh Tam, Phan Thanh Cong, Nguyen Quoc Viet Hung, Karl Aberer, Bela Stantic

PII: S1566-2535(18)30252-5

DOI: 10.1016/j.inffus.2018.07.009

Reference: INFFUS 1001

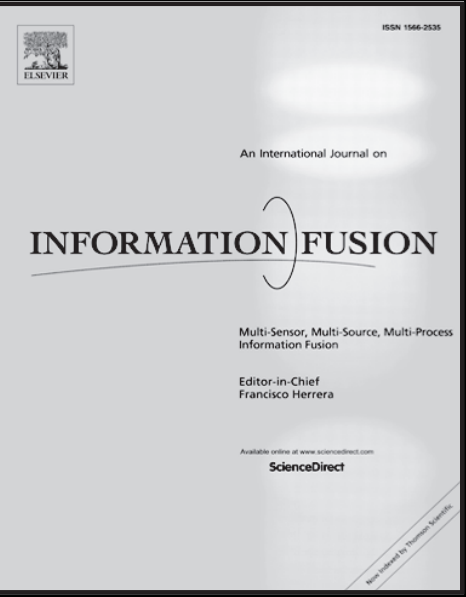

To appear in:

Information Fusion

Received date:

8 April 2018

Revised date:

27 July 2018

Accepted date:

30 July 2018

Please cite this article as: Nguyen Thanh Tam, Phan Thanh Cong, Nguyen Quoc Viet Hung, Karl Aberer, Bela Stantic, Maximal Fusion of Facts on the Web with Credibility Guarantee, Information Fusion (2018), doi: 10.1016/j.inffus.2018.07.009

This is a PDF file of an unedited manuscript that has been accepted for publication. As a service to our customers we are providing this early version of the manuscript. The manuscript will undergo copyediting, typesetting, and review of the resulting proof before it is published in its final form. Please note that during the production process errors may be discovered which could affect the content, and all legal disclaimers that apply to the journal pertain. 